\title{
Novel mutations of epidermolysis bullosa identified using whole- exome sequencing in Indonesian Javanese patients
}

\author{
Suci Widhiati ${ }^{1,2}$, Retno Danarti ${ }^{3}$, Niken Trisnowati ${ }^{3}$, Dewajani Purnomosari ${ }^{2,4}$, Tri Wibawa ${ }^{2,5}$, \\ Hardyanto Soebono ${ }^{2,3, *}$ \\ ${ }^{1}$ Department of Dermatology and Venereology, Faculty of Medicine, Universitas Sebelas Maret, Surakarta, Indonesia; \\ ${ }^{2}$ Doctoral Study Program, Faculty of Medicine, Public Health and Nursing Universitas Gadjah Mada, Yogyakarta, Indonesia; \\ ${ }^{3}$ Department of Dermatology and Venereology, Faculty of Medicine, Public Health and Nursing, Universitas Gadjah Mada, Yogyakarta, Indonesia; \\ ${ }^{4}$ Department of Histology and Cell Biology, Faculty of Medicine, Public Health and Nursing, Universitas Gadjah Mada, Yogyakarta, Indonesia; \\ ${ }^{5}$ Department of Microbiology, Faculty of Medicine, Public Health and Nursing, Universitas Gadjah Mada, Yogyakarta, Indonesia.
}

SUMMARY Epidermolysis bullosa (EB) is a group of inherited blistering skin diseases known to have heterogenicity of phenotypes and genotypes. There are four main types of EB: simplex, junctional, dystrophic, and Kindler syndrome, which are further classified into 34 distinct subtypes. Twenty different gene mutations are responsible for the loss of function and integrity of the basal membrane zone. In limited-resource settings such as Indonesia, diagnoses of hereditary skin disease often rely on clinical features. This limitation was managed by using the Clinical Diagnostic Matrix EB for clinical diagnosis support and whole-exome sequencing for genetic analysis. This study is the first wholeexome sequencing analysis of Javanese Indonesian patients with EB. The genetic analysis from four patients with EB identified all novel mutations unreported in the dbSNP database. There are Kindler syndrome with FERMT1 frameshift mutation in exon 4, at c.388A (p.I130fs), which causes truncated protein; junctional EB generalized intermediate (JEB-GI) subtype with missense mutation at LAMB3 gene position c.A962C (p.H321P); and recessive dystrophic EB (RDEB) a missense mutation at COL7A1 gene position c.G5000T (p.G1667V). The whole-exome sequencing was further verified by Sanger sequencing. The new mutations' finding is possibly due to the limited genetic database in the Malayo-Polynesian ethnic group. Indonesia has hundreds of ethnic groups, and the Javanese is the largest ethnic group that populates Indonesia. Genetic data of these ethnic groups is important to be established in the international genetic database. This combination of clinical diagnostic and genetic analysis tools with whole-exome sequencing confirmed the challenging diagnosis of epidermolysis bullosa.

Keywords clinical diagnostic matrix, Kindler syndrome, junctional epidermolysis bullosa, dystrophic epidermolysis bullosa, Malayo-Polynesian ethnic group

\section{Introduction}

Epidermolysis bullosa (EB) is an inherited skin disorder characterized by flaky skin and blisters after light trauma or friction. The wounds tend to occur repeatedly and may become chronic, which causes high morbidity. This disease's clinical manifestations vary, ranging from local wounds on the hands and feet to generalized blisters or erosion and failure to thrive (l).

Epidermolysis bullosa comprises four major subtypes: EB simplex, junctional EB (JEB), dystrophic EB (DEB), and Kindler syndrome (KS). Each subtype is classified based on the molecular structure mutation in the dermis-epidermis (2). Before high-throughput next-generation sequencing (NGS) was developed, diagnosis of $\mathrm{EB}$ required an invasive procedure such as skin biopsy followed by microscopic examination and/ or direct immunofluorescence to determine the mutation locations and finally, the results were confirmed by Sanger sequencing (SS) as the gold standard of diagnosis (3).

Caused by mutations in 20 different genes, EB involves the loss of function and integrity of the basal membrane zone structure. These genes code structured proteins in the intermediate filaments (keratin 5 and 14), focal adhesions, and desmosome complexes (desmoplakin, plakophilin, and plakoglobin). They are also vital to form intraepidermal adhesions and the 
dermo-epidermal anchoring complex in the basement membrane zone of the mucous membrane and skin (integrin 64, collagen type XVII, laminin 332, collagen type VII, integrin three subunits, and kindlin-1) (4). Unfortunately, SS can only test single or a few genes at a time; thus, it is time-consuming and costly to do SS in cases when multiple genes need to be tested. Recently, direct procedures with NGS or targeted NGS have been performed to overcome the previous procedure's limitations. NGS screens all the suspected genes involved in EB using a single sample (3).

The NGS technology can simultaneously sequence the peptides/proteins coded by a known gene in the genome called whole-exome sequencing (WES). Even though WES can only sequence $1.5 \%$ of the entire human genome, $85 \%$ of the pathogenic mutations occur in regions encoded by protein, where WES is preferable. In recent years, the decreased cost of WES has developed a potential preference to use WES as a clinical practice (5).

In a limited-resource setting such as Indonesia, EB and EB subtypes' diagnosis depends on clinical findings and hematoxylin-eosin staining biopsy results. Electron microscopic and immunofluorescence examinations are not routinely done because they are rarely available in many centers. Thus, this condition implies a holistic approach to care for patients with EB. WES sequencing for analyzing all genes simultaneously to find the pathogenic variants will have a tremendous impact on both the novel search for disease gene discoveries and the efficient diagnosis of known genetic diseases in Indonesia.

Novel mutations or novel variants can be identified when they are not present in unrelated healthy control samples that were obtained from the exome sequence databases Exome Aggregation Consortium (ExAC) browser (https://gnomad.broadinstitute.org) and other databases such as dbSNP, 1000 Genomes Project (http:// www.1000genomes.org), and Genome-Wide Association Studies (GWAS).

Javanese comprises the most prominent traits in Indonesian people who populate Java Island, and genetic testing with WES for the inherited disorder to the researchers' knowledge has never been done before in Javanese Indonesians. The prevalence of EB in Indonesia remains unknown. Based on case reports between 2013-2020, there were nine cases in Javanese traits, which is less than expected. Diana et al. reported around $31 \mathrm{~EB}$ cases in Indonesia in 2018 based on the Epidermolysis Bullosa Community Indonesia database (6). Even though genetic variants have been reported extensively in other populations, a study in the Javanese Indonesian population is lacking.

This study explores the use of WES to diagnose EB cases in Javanese Indonesians. This study highlights the first-tier use of WES to analyze genetic variation in Javanese EB cases. It will hopefully become a standard diagnostic tool for EB and other genetic disorders in Indonesia.

\section{Materials and Methods}

The clinical subtype diagnosis of EB was performed using a clinical matrix diagnostic tool (7), consisting of nine of the most often identified subtypes of EB. From these, the clinicians chose the most appropriate clinical findings that matched the available matrix.

DNA extraction from patients, parents, and healthy controls were obtained after informed consent for genetic analyses and medical information disclosure. Ethical clearance was completed following the Declaration of Helsinki with approval from the Universitas Gadjah Mada Medical and Health Research Ethics Committee. Four patients with EB, five unaffected parents, and ten healthy controls were included in this study.

Genomic DNA was purified using the AllPrep DNA/ RNA Mini Kit (QIAGEN, Hilden, Germany). The concentration and quality of genomic DNA (gDNA) extracted from peripheral blood were examined with NanoDrop ND-100 (Thermo Scientific) and agarose gel electrophoresis.

Whole-exome sequencing was performed at Novogene (Hongkong, China). DNA was captured using the Agilent SureSelect Human All Exon kit and sequenced on an Illumina HiSeq platform. The sequence was aligned to the human genome reference sequence (hg38) using the Burrows-Wheeler Aligner (v0.7.8-r455). Duplicate reads were removed using Picard (v1.111), single nucleotide polymorphisms (SNPs) and Indels (insertions and deletions) were identified (SAMtools v1.0). The detected variants were then annotated using ANNOVAR (8). We defined database annotation variants with reported allele frequencies of $0.5 \%$ or greater in one of the following databases: dbSNP, 1000 Genomes Project (http://www.1000genomes.org), Genome-Wide Association Studies (GWAS), and Exome Aggregation Consortium (ExAC). We focused on variants of known causal genes in EB diseases, including KRT5, KRT14, DST, EXPH5, DSP, PLEC, LAMA3, LAMB3, ITGA3, LAMC3, ITGB4, ITGA6, JUP, COL7A1, COL17A1, EXPH5, FERMT1, PKP1, and TGM5. From those variants, we filtered deleterious predicted variants according to the following criteria: frameshift, in-frame insertion and deletions, stop codon changes, missense unless predicted innocuous by SIFT, polyphen-2, Mutation Taster, likelihood ratio test (LRT), Mutation Assessor, and Functional Analysis Through Hidden Markov Models (FATHMM).

The SS was performed to confirm the pathogenic variants in the patient, parents, and the control group using an ABI PRISM ${ }^{\circledR}$ BigDye Terminator Cycle Sequencing Kit v3.1 (Applied Biosystems; Thermo Fisher Scientific, Inc.). The primer sequences of each of the suspected pathogenic variant genes 
FERMT1 and $L A M B 3$ were chosen based on previous studies $(9,10)$, the COL7A1 exon 53-54 were modified from Cristianto et al. in 1997 with forward 5'CCTTGAGAACTGCTTGCTTC3' and reverse 5'GAAGATTGGGAGGGTTTAGC3' (11). The sequence reads using 4peaks application (https://nucleobytes. com/4peaks).

To identify the novelty of EB variants in Indonesia, the literature search was conducted using PubMed and Google Scholar with the term "epidermolysis bullosa" "southeast Asia" "Indonesia" "Javanese" and the full texts of retrieved literature were read carefully. Personal communication with clinicians was also established to find EB cases in Central Java and Yogyakarta. Novel variants are considered when the variant annotation is not found in the databases: dbSNP, 1000 Genomes Project (http://www.1000genomes.org), GenomeWide Association Studies (GWAS), and the Exome Aggregation Consortium (ExAC).

\section{Results and Discussion}

Four patients with clinically confirmed EB were included in this study. The clinical diagnosis was made with support from clinical matrix diagnostic tools, and all patients underwent genetic analysis. Two patients are not related, but Cases 2 and 3 were siblings. Two patients are male, and two are female with an age range of 10 to 22 years. Three from 4 subjects were missense mutations; one subject was a frameshift mutation. The subtypes of EB were varied from Kindler syndrome, JEB-GI, and RDEB-GS. All patients are genetically of Javanese ethnicity.

Each of the pathogenic EB variants was found to be deleterious by its rare or low frequency. We documented novel mutations in all subjects with WES (Table 1). The targeted EB variants in our study were not reported in the dbSNP, GWAS study, 1000 Genomes Project (released in August 2015) nor in the ExAC. Evaluation of 10 unrelated control individuals by SS failed to demonstrate all the mutations found in this study. Thus, we identified pathogenic novel mutations in the FERMT1, LAMB3, and COL7A1 genes, which confirmed Kindler syndrome, JEB-GI, and RDEB, respectively.

Clinical WES is a valuable diagnostic tool to pinpoint rare genetic alterations in an unbiased and efficient way to confirm a diagnosis despite extensive testing and workup. The use of WES as a first-tier test would substantially reduce the time to diagnosis, and it is only a half to one-quarter the cost of traditional testing (12). WES has a consistently higher diagnostic yield than routine clinical SS. However, the Sanger method is still considered the "gold standard" for clinical DNA sequencing. Nevertheless, SS has limitations because it can only test single or a few genes at a time and becomes costly when multiple genes are tested before reaching a diagnosis (13). Hamilton et al. in 2016 demonstrated that WES has high concordance with SS with $97.3 \%$ of variants concordant (13).

However, WES has some limitations in poor targeting by the exome capture kit, high guaninecytosine (GC) content, and the presence of repetitive sequences, leading to inadequate coverage or sequencing inaccuracy. The exome capture kit used to define allele frequencies in our study was using the 1000 Genomes Project, GWAS, ExAC, and dbSNP. Unfortunately, none of the commercial arrays for SNPs databases were created from polymorphism data generated in Southeast Asian Malays. In the future, it is essential to develop population-specific databases as a country-based molecular variation database since different genetic backgrounds may have different susceptibility to the haploid in the sufficiency of variants or mutations. SNPS may contribute to the genetic disorder, or they may be in linkage disequilibrium with other causal variants and mutations (14).

Indonesia, located in Southeast Asia, comprises more than 17,000 islands, including the five biggest islands, which are Sumatra, Java, Borneo (Kalimantan),

Table 1. EB associated variants identified with whole-exome sequencing in-silico analysis

\begin{tabular}{|c|c|c|c|c|}
\hline Characteristic variant & Case 1 & Case 2 & Case 3 & Case 4 \\
\hline Annotation & Indels & SNP & SNP & SNP \\
\hline \multirow[t]{3}{*}{ Gene name } & FERMT1 & $L A M B 3$ & $L A M B 3$ & COL7A1 \\
\hline & NM_017671 & NM_001017402 & NM_001017402 & NM_000094 \\
\hline & exon 4 & exon 9 & exon 9 & exon 54 \\
\hline Amino acid change & p.I130fs & p.H321P & p.H321P & p.G1667V \\
\hline cDNA & c.388dupA & c.A962C & c.A962C & c.G5000T \\
\hline Known variant & Novel $^{*}$ & Novel $^{*}$ & Novel $^{*}$ & Novel $^{*}$ \\
\hline \multirow[t]{2}{*}{ SIFT } & - & Deleterious & Deleterious & Deleterious \\
\hline & & $(0.002)$ & $(0.002)$ & $(0)$ \\
\hline Polyphen2 & - & Probably damaging (0.999) & Probably damaging (0.999) & Probably damaging (1.0) \\
\hline Mutation taster & - & Disease-causing & Disease-causing & Disease-causing \\
\hline LRT & - & Deleterious & Deleterious & Deleterious \\
\hline FATHMM & - & Tolerated (-0.2) & Tolerated (-0.2) & Tolerated (-4.62) \\
\hline
\end{tabular}

"Variant not found in dbSNP, 1000 Genomes Project, GWAS, ExAC, nor ESP database. 
Sulawesi, and Papua. Indonesia is the world's $4^{\text {th }}$ mostpopulous country with over 267 million people, and Java is the most populous island. Indonesia consists of hundreds of distinct native ethnic and linguistic groups, with the largest being Javanese (https://en.wikipedia.org/ wiki/Indonesia). Our study is located in Central Java and Yogyakarta, which is mostly populated by people with Javanese ancestry.

Case 1, a 12-year-old boy with clinical manifestation of skin brittle, poikiloderma, meatal stenosis, aphthous ulcer, and history of hernia inguinal (Figure 1). On genetic analysis, we identified a homozygous mutation in the location of 389 FERMT1, an insertion of A-base, that converts an isoleucine codon (ATT) at codon 130 to asparagine codon (AAT) and leads to a premature termination codon (PTC) at codon 132 (p.I130fs). The parents were heterozygous for the mutation and were considered obligate carriers. The location of the
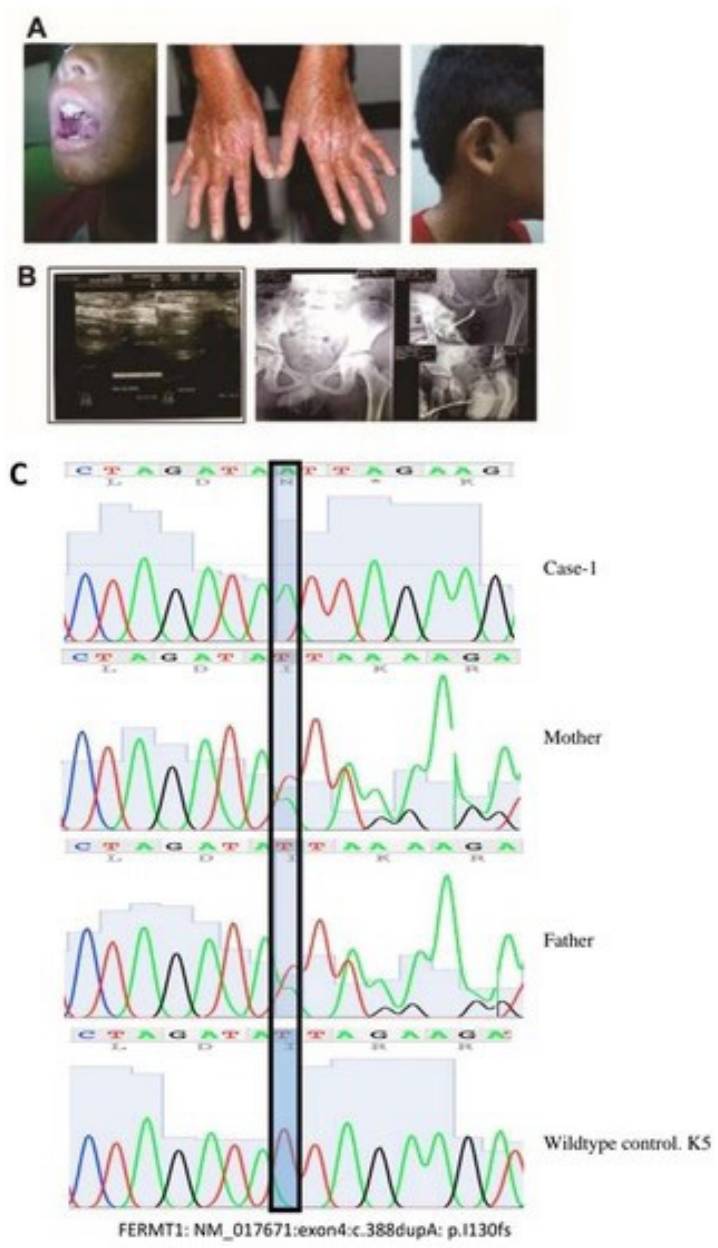

Figure 1. Clinical manifestations of Kindler syndrome and electropherogram of FERMT1 mutation. (A) Poikilodermas on the face and neck and hands; aphthous ulcer of buccal gingiva; atrophic skin on hands; (B) Ultrasonography imaging: bowel defect in the left inguinal region during Valsalva test; contrasted urethrography image: stenosis of the urethra. (C) A frameshift mutation of FERMT1 gene duplicated $\mathrm{T}$ base resulting in changes of amino acid and premature stop codon; heterozygous mutation on father and mother; and normal wildtype control. mutation is at the F1 domain, which is considered highly conserved. We confirmed the diagnosis of Kindler syndrome. The mutation of c.388dupA p.I130fs is the first reported in the literature. The duplication of FERMT1 in exon 4 has been reported previously (15), but the correlation between duplication and severity of the clinical findings is still unclear. The mutation in our study is in the $\mathrm{F} 1$ segment of FERM, which is predicted to cause loss of the FERM domains of the protein, thus resulting in the detached adhesin bond and membrane matrix (16). Furthermore, the isoleucine protein in the F1 domain is a highly conserved domain (17). This mutation appears to contribute to the pathogenesis of several organs, not only manifesting in skin atrophy and poikiloderma but also including dysfunction of the oral, intestinal, and urinary systems.

Cases 2 and 3 are siblings with various clinical manifestations (Figure 2). The chronic insole ulcers
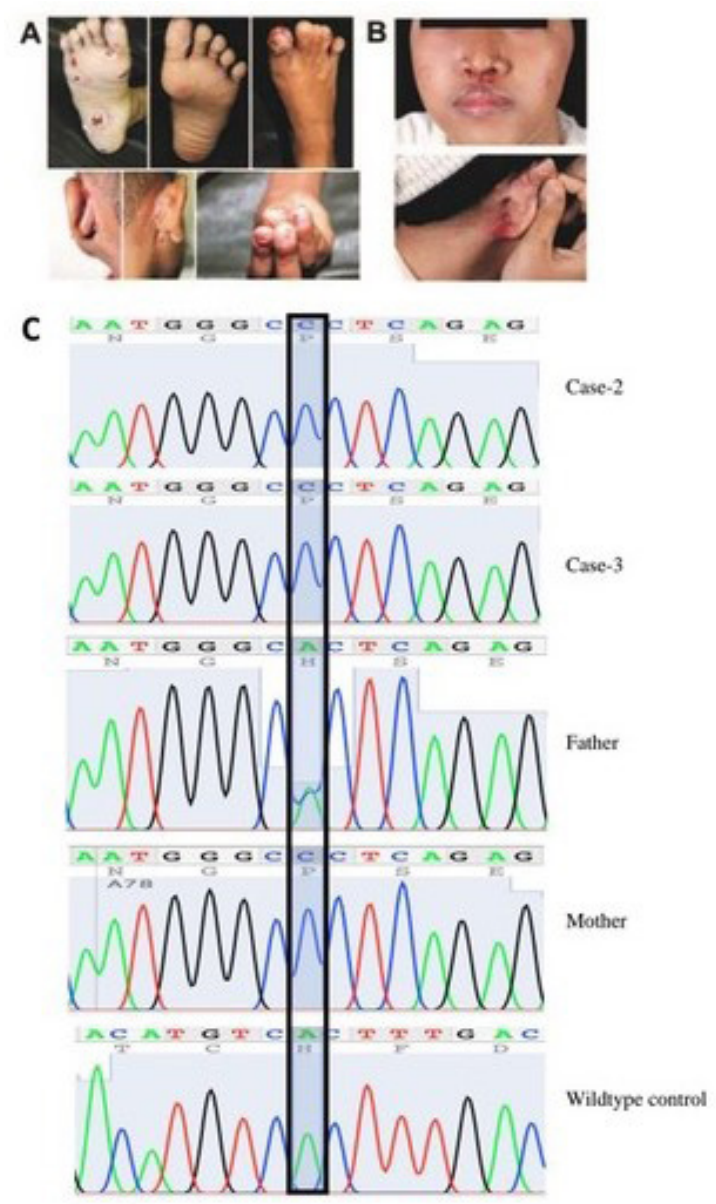

LAMB3: NM_001017402:exon9:C.A962C:p.H321P,

Figure 2. Clinical manifestations of JEB and electropherogram of LAMB3 mutation. (A) Chronic ulcer on the insole, back of the ear, and hand fingers of Case 2; (B) Chronic ulcer on the nose philtrum and back of the ear of Case 3; (C) sequencing of LAMB3 of Case 2 and Case 3. A single-base substitution of c. $962 \mathrm{~A}>\mathrm{C}$ in brother (Case 2) and sister (Case 3) was observed, and it was also apparent in mother while father is heterozygous carrier, and the wildtype control shows no mutation. 


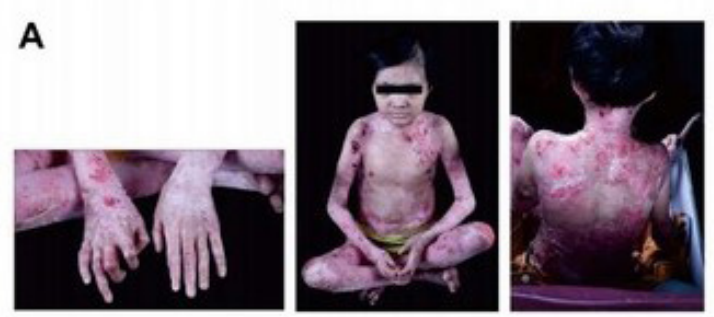

B

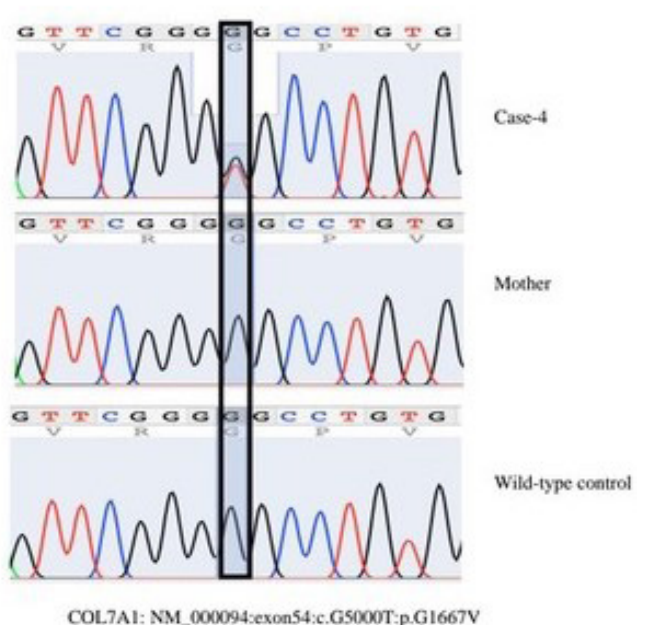

Figure 3. Clinical manifestations of RDEB and electropherogram of $C O L 7 A 1$ mutation. (A) skin erosion and right point finger contracture in Case 4; and (B) heterozygous missense mutation at c.5000G $>\mathrm{T}$, which was not shown in wildtype control nor the mother.

and finger ulcers were seen in the 10-year-old younger brother, but not in his sister, instead, a chronic ulcer at the philtrum was seen. Both of them have ulcers behind their ear, and unsurprisingly they have the same homozygous mutation in the location of 962 LAMB3 A $>$ C. The histidine codon (CAC) converts to a proline residue $(\mathrm{C} \underline{C})$. The mother also has the same homozygous variant, but interestingly she did not have any clinical manifestations. The father has a heterozygous variant in the same allele. The diagnosis confirmed junctional EB intermediate. Mutation of the $L A M B 3$ gene is the hallmark of JEB, the critical encoding component of the hemidesmosome-anchoring filament complex that links the keratin cytoskeleton to the lamina densa of the dermo-epidermal junction (18). In the majority of the JEB cases, $80 \%$ of mutations predominantly affect the $L A M B 3$ gene. However, the clinical spectrum is highly variable depending on the mutation's type and position (19). We identified siblings with the same mutation of $L A M B 3$ at the same loci c.A962C (p.H321P), but they demonstrated different phenotypes. Other polymorphisms in each person may correlate with various clinical manifestations. However, this possible explanation needs to be addressed in future studies.

In Case 4, the result of WES and SS had high concordance. The WES presented a mutation in the
$\mathrm{G}>\mathrm{T}$ exon $54 \mathrm{COL7A1}$, and SS reported a heterozygous mutation at codon 5000 in the affected child (Figure 3). However, we did not find the variant in the mother nor wildtype control. Unfortunately, paternal DNA cannot be defined. Thus, we diagnosed the patient with recessive dystrophic epidermolysis bullosa. COL7A1 is responsible for dystrophic EB with dominant and recessive genetic patterns, though genetic analyses of the COL7A1 gene in affected individuals revealed that most mutations detected are recessive DEB (RDEB) (20). The majority of severe RDEB cases are caused by PTC mutations resulting from nonsense, frameshift, or splice-site mutations on both COL7A1 alleles, which result in either nonsense-mediated decay of the mRNA or truncated polypeptides, and the result is unable to assemble the functional protein and the mRNA degraded within the cell. Milder cases of RDEB are often caused by compound heterozygous mutations: one PTC mutation and a missense mutation (21). Our patient had a heterozygous missense mutation of COL7A1 at exon 54 substitution $5000 \mathrm{G}>\mathrm{T}$ that substitutes glycine for valine. Mallipeddi et al. in 2003 pointed out the need to search for further mutations when they found glycine substitution in sporadic cases (22). However, our case did not reveal other mutations on COL7A1. The heterogeneous mutations in our case caused disease phenotypes and led to moderate-to-severe DEB forms, unlike in other articles, which need to be further examined (23).

Murata et al. in 2003 demonstrated that the presence of recurrent mutations could be classified into specific ethnic groups and worldwide mutations (20). Our four cases revealed novel mutations that were not recorded in any public SNP database (dbSNP). Our novel finding is probably because of the limited number of EB Malay traits in reported articles, resulting in a lack of identified recurrent specific ethnic group mutations in Malay ancestry. Abu Sa'd et al. in 2006 also stated that diagnostic strategies based upon molecular epidemiological features that are determined in Western origin populations might not apply to Middle Eastern populations (24). However, two studies reported two Sundanese Indonesian families with dystrophic EB. They reported the mutations in $C O L 7 A 1$ at c.6218G>A; pGly2073Asp, c.5945G.T; pGly1982Val and c.6253G $>$ T; p.Gly2085Trp $(25,26)$. We did not find other articles associated with EB genetic examination in Indonesian traits.

As a limitation, the number of subjects was too small to analyze a high diagnostic yield in WES compared to standard genetic testing. The low incidence of cases may hamper subject recruitment.

In conclusion, we demonstrated a novel mutation in Kindler syndrome on c.388A (p.I130fs), junctional epidermolysis bullosa intermediate on c.A962C (p.H321P), and recessive dystrophic epidermolysis bullosa on $5000 \mathrm{G}>\mathrm{T}$ (p.G1667V) as Javanese traits. In 
these cases, practical genetic analysis was best performed using whole-exome sequencing, a less invasive genetic diagnostic tool, with a reduced time to diagnosis.

\section{Acknowledgements}

Thanks to Willa Damayanti, Indah Julianto, and Endra Yustin at the Pediatric Dermatology division Faculty of Medicine Universitas Sebelas Maret for case discussion; The staff of Klinik Bahasa in the Office of Research and Publication, Faculty of Medicine, Public Health and Nursing, Universitas Gadjah Mada (FKKMK UGM); and Sri Fatmawati for technical support at the Laboratorium Terpadu, FKKMK UGM. Thank you to all patients and their families who participated in this study.

Funding: The work was supported by Rekognisi Tugas Akhir Universitas Gadjah Mada, Yogyakarta, Indonesia.

Conflict of Interest: The authors have no conflicts of interest to disclose.

\section{References}

1. Fine JD. Inherited epidermolysis bullosa. Orphanet J Rare Dis. 2010; 5:12.

2. Fine JD, Bruckner-Tuderman L, Eady RA, et al. Inherited epidermolysis bullosa: updated recommendations on diagnosis and classification. J Am Acad Dermatol. 2014; 70:1103-1126.

3. Lucky AW, Dagaonkar N, Lammers K, Husami A, Kissell D, Zhang K. A comprehensive next-generation sequencing assay for the diagnosis of epidermolysis bullosa. Pediatr Dermatol. 2018; 35:188-197.

4. Has C, Fischer J. Inherited epidermolysis bullosa: New diagnostics and new clinical phenotypes. Exp Dermatol. 2019; 28:1146-1152.

5. Takeichi T, Liu L, Fong K, Ozoemena L, McMillan JR, Salam A, Campbell P, Akiyama M, Mellerio JE, McLean WH, Simpson MA, McGrath JA. Whole-exome sequencing improves mutation detection in a diagnostic epidermolysis bullosa laboratory. Br J Dermatol. 2015; 172:94-100.

6. Diana IA. DEBRA Indonesia, chalenges and inovation. Group PDS, ed. PERDOSKI, Surabaya, 2018.

7. Yenamandra VK, Moss C, Sreenivas V, Khan M, Sivasubbu S, Sharma VK, Sethuraman G. Development of a clinical diagnostic matrix for characterizing inherited epidermolysis bullosa. Br J Dermatol. 2017; 176:16241632.

8. Wang K, Li M, Hakonarson H. ANNOVAR: functional annotation of genetic variants from high-throughput sequencing data. Nucleic Acids Res. 2010; 38:e164.

9. McGrath JA, Kivirikko S, Ciatti S, Moss C, Christiano AM, Uitto J. A recurrent homozygous nonsense mutation within the LAMA3 gene as a cause of Herlitz junctional epidermolysis bullosa in patients of Pakistani ancestry: evidence for a founder effect. J Invest Dermatol. 1996; 106:781-784.

10. Siegel DH, Ashton GH, Penagos HG, et al. Loss of kindlin-1, a human homolog of the Caenorhabditis elegans actin-extracellular-matrix linker protein UNC112, causes Kindler syndrome. Am J Hum Genet. 2003; 73:174-187.

11. Christiano AM, Hoffman GG, Zhang X, Xu Y, Tamai Y, Greenspan DS, Uitto J. Strategy for identification of sequence variants in COL7A1 and a novel 2-bp deletion mutation in recessive dystrophic epidermolysis bullosa. Hum Mutat. 1997; 10:408-414.

12. Niguidula N, Alamillo C, Shahmirzadi Mowlavi L, Powis Z, Cohen JS, Farwell Hagman KD. Clinical whole-exome sequencing results impact medical management. Mol Genet Genomic Med. 2018; 6:1068-1078.

13. Hamilton A, Tetreault M, Dyment DA, Zou R, Kernohan K, Geraghty MT, Consortium FC, Care4Rare Canada C, Hartley T, Boycott KM. Concordance between wholeexome sequencing and clinical Sanger sequencing: implications for patient care. Mol Genet Genomic Med. 2016; 4:504-512.

14. Halim-Fikri H, Etemad A, Abdul Latif AZ, et al. The first Malay database toward the ethnic-specific target molecular variation. BMC Res Notes. 2015; 8:176.

15. Has C, Castiglia D, del Rio M, Diez MG, Piccinni E, Kiritsi D, Kohlhase J, Itin P, Martin L, Fischer J, Zambruno G, Bruckner-Tuderman L. Kindler syndrome: extension of FERMT1 mutational spectrum and natural history. Hum Mutat. 2011; 32:1204-1212.

16. Lai-Cheong JE, Parsons M, Tanaka A, Ussar S, South AP, Gomathy S, Mee JB, Barbaroux JB, Techanukul T, Almaani N, Clements SE, Hart IR, McGrath JA. Lossof-function FERMT1 mutations in kindler syndrome implicate a role for fermitin family homolog-1 in integrin activation. Am J Pathol. 2009; 175:1431-1441.

17. Lu S, Wang J, Chitsaz F, et al. CDD/SPARCLE: the conserved domain database in 2020. Nucleic Acids Res. 2020; 48:D265-D268.

18. Milenkovic D, Chaffaux S, Taourit S, Guerin G. A mutation in the LAMC2 gene causes the Herlitz junctional epidermolysis bullosa (H-JEB) in two French draft horse breeds. Genet Sel Evol. 2003; 35:249-256.

19. Yenamandra VK, Vellarikkal SK, Kumar M, Chowdhury MR, Jayarajan R, Verma A, Scaria V, Sivasubbu S, Ray SB, Dinda AK, Kabra M, Kaur P, Sharma VK, Sethuraman G. Application of whole exome sequencing in elucidating the phenotype and genotype spectrum of junctional epidermolysis bullosa: A preliminary experience of a tertiary care centre in India. J Dermatol Sci. 2017; 86:30-36.

20. Murata T, Masunaga T, Ishiko A, Shimizu H, Nishikawa T. Differences in recurrent COL7A1 mutations in dystrophic epidermolysis bullosa: ethnic-specific and worldwide recurrent mutations. Arch Dermatol Res. 2004; 295:442447.

21. Yan Y, Meng Z, Hao S, Wang F, Jin X, Sun D, Gao H, Ma $X$. Five novel COL7A1 gene mutations in three Chinese patients with recessive dystrophic epidermolysis bullosa. Ann Clin Lab Sci. 2018; 48:100-105.

22. Mallipeddi R, Bleck O, Mellerio JE, Ashton GH, Eady RA, McGrath JA. Dilemmas in distinguishing between dominant and recessive forms of dystrophic epidermolysis bullosa. Br J Dermatol. 2003; 149:810818.

23. Kern JS, Kohlhase J, Bruckner-Tuderman L, Has C. Expanding the COL7A1 mutation database: novel and recurrent mutations and unusual genotypephenotype constellations in 41 patients with dystrophic 
epidermolysis bullosa. J Invest Dermatol. 2006; 126:1006-1012.

24. Abu Sa'd J, Indelman M, Pfendner E, et al. Molecular epidemiology of hereditary epidermolysis bullosa in a Middle Eastern population. J Invest Dermatol. 2006; 126:777-781.

25. Diana IA, Gondokaryono SP, Dwiyana RF, Rahardja JI, Yogya Y, Gunawan H. Intrafamilial diversity of clinical severity of epidermolysis bullosa: case series of three generations. The Open Dermatology Journal. 2019; 13:37.

26. Bishnoi P, Ng YZ, Wei H, Tan E-C, Lunny DP, Wong XFCC, Fon LK, Gondokaryono SP, Diana IA, Common JEA, Koh MJA, Lane EB. Self-improving dystrophic epidermolysis bullosa: First report of clinical, molecular, and genetic characterization of five patients from Southeast Asia. Am J Med Genet. 2021; 185:625-630.

Received November 26, 2020; Revised February 25, 2021; Accepted March 5, 2021.

*Address correspondence to:

Hardyanto Soebono, Department of Dermatology and Venereology, Faculty of Medicine, Public Health and Nursing, Universitas Gadjah Mada, Gedung Radiopoetro 1t3, J1. Farmako Sekip Utara, Sleman, Yogyakarta 55281, Indonesia

E-mail: hardyanto@ugm.ac.id

Released online in J-STAGE as advance publication March 18, 2021. 